\title{
Quantitative Evaluation of Reserve Projects Based on the Grid Contribution
}

\author{
Zifa Liu ${ }^{1, *}$, Dong Peng ${ }^{2}$, Xuyang Wang ${ }^{1}$, Zhe Zhang ${ }^{1}$, Yawei Xue ${ }^{2}$ and Hui $\mathrm{Li}^{2}$ \\ ${ }^{1}$ North China Electric Power University, Beijing, China \\ ${ }^{2}$ State Grid Economic and Technological Research Institute CO.LTD, Beijing, China \\ ${ }^{*}$ Corresponding author
}

\begin{abstract}
The comprehensive evaluation of electric construction project is one of the main tasks in the early stage of the project. In order to accurately embody the nature, content, and scale of the reserve projects, a quantitative evaluation method for reserve projects based on the contribution of power grid is proposed in this paper. An evaluation index system and its quantitative calculation method are established, And the optimal weight of each index is determined by utilizing the combination assigning method and particle swarm optimization algorithm. Then the contribution evaluation model of power grid reserve project is constructed based on the contribution and project investment. The dynamic sorting method for power grid reserve projects is determined by analyzing the coupling relationship and decoupling method in the reserve projects. Finally, the distribution network expansion projects in a certain city are taken as an example, and the results are presented to illustrate the usefulness of proposed method.
\end{abstract}

Keywords-reserve project; quantitative evaluation; combination weighting method; particle swarm optimization algorithm

\section{INTRODUCTION}

The evaluation and selection of power grid reserve project is the last threshold for power grid investment management, which is the key to ensure the precision level and investment benefit of power grid investment. The evaluation of power projects can be divided into pre-evaluation, mid-term evaluation and post-evaluation. The post-evaluation can analyze the subjective and objective reasons for the success or failure of investment activities, and the projects can be evaluated fairly and objectively.

Fuzzy mathematics is applied to the multi-objective comprehensive evaluation to form a fuzzy comprehensive evaluation model in Ref [1]. According to the subordination theory, the qualitative evaluation is transformed into quantitative evaluation to solve various non-deterministic problem. The technical post-evaluation, financial postevaluation, national economic evaluation and affective postevaluation of power investment projects are analyzed from the perspective of power grid projects, and a post-evaluation method system for power investment projects is established in Ref [2]. A cost-benefit analysis theory is applied to the methodology and mechanism for post-evaluation of power projects in Ref [3]. Moreover, the index system and evaluation method for the construction evaluation, performance evaluation, environmental impact assessment, continuous evaluation and comprehensive evaluation of power investment projects are studied in Ref[4].

Many achievements have been made in the evaluation of Chinese power grid construction project, but with the changes of the power industry, existing evaluation methods have some shortcomings[5]:(1) The power grid construction projects only focus on the feasibility and necessity analysis from the perspective of technology and safety, however, the analysis of the economic perspective is weakened.(2) The evaluation is inaccurate for the lack of the analysis and quantification of uncertain factors in the construction of grid projects.(3) The evaluation methods and dynamic optimization management of the grid reserve projects need to be further improved.

According to these problems, a quantitative evaluation method for reserve projects based on the contribution of grid performance is proposed in this paper. Compared to existing literature, contributions of this work include: (1) An evaluation index system and its quantitative calculation method are established, and its optimal weight of each index is determined by utilizing the combination assigning method and particle swarm optimization algorithm. (2) Then the contribution evaluation model of power grid reserve project is constructed based on the contribution and project investment; (3) The dynamic sorting method for power grid reserve projects is determined by analyzing the coupling relationship and decoupling method in the reserve projects.

\section{Evaluation INDEX System AND CALCUlation OF RESERVE PROJECTS}

According to the principle of systematicness, scientificity, practicability, availability, and comparability [6], considering the existing evaluation indexes of the power grid construction project, the evaluation indexes needed for this paper are extracted from the following five aspects.

\section{A. Security Indexes}

\section{1) Satisfaction rate of $\mathrm{N}-1$ criterion}

The calculation formula for the satisfaction rate of $\mathrm{N}-1$ criterion is as follows:

$$
\eta_{N-1}=\frac{N_{N-1}}{N_{\text {total }}}
$$


where $\eta_{N-1}$ represents the satisfaction rate of $\mathrm{N}-1$ criterion; $N_{N-1}$ represents the component number of satisfying the $\mathrm{N}-1$ criterion; $N_{\text {total }}$ is the total number of components.

\section{2) Load transfer capacity}

The load transfer capability is obtained from the transfer load of the corresponding substation due to feeder fault.

$$
P_{R L}=\sum_{m=0}^{m<M} \beta_{m} L_{m}, \quad M<N
$$

where $P_{R L}$ represents the load transfer capability; $M$ represents the number of recovered transformers; $N$ represents the number of transformers; $\beta$ represents the importance degree of the load $m$, the range of values is $[0,1], L$ represents the power of the load $m$.

\section{3) Line connection rate}

The line connection rate in the distribution network is defined as:

$$
\eta_{\text {connect }}=\frac{L_{\text {connect }}}{L_{\text {total }}} \times 100 \%
$$

where, $\eta$ connect represents the line connection rate, $L$ connect represents the number of connected lines, $L$ total represents the total number of lines.

\section{B. Transmission Capacity}

The transmission capacity is the difference between the total ingress capacity and egress capacity of the current voltage level in the area [7]. This paper uses probabilistic statistics method to calculate the corresponding available transmission capacity(ATC) for each sampling sample. After accumulating a sufficient number of samples, the ATC probability statistical indexes of the transmission system can be obtained [8-9].

Assuming that $\mathrm{N}$ represents the total number of samples, and ATC(xi) represents the ATC of the i-th sample xi. according to the probability theory, the expected value of the ATC E(ATC) is calculated as follows:

$$
E(A T C)=\frac{1}{N} \sum_{i=1}^{N} A T C\left(x_{i}\right)
$$

\section{1) Power supply capacity}

For a whole distribution network with multiple interconnected substations, its power supply capacity of satisfy the $\mathrm{N}-1$ criteria is calculated as follows:

$$
\max P=\sum_{j}\left(R_{j} \cdot T_{j}\right)+\sum_{i} L_{i}
$$

where $P$ represents the power supply capability of the distribution system, $R_{j}$ represents the rated capacity of the main transformer $\mathrm{j}, T_{j}$ is the load rate of the main transformer $\mathrm{j}$, and $L_{i}$ is the ATC of the $\mathrm{i}$-th line.

\section{Operation Indexes}

\section{1) Grid loss rate}

It is difficult to accurately calculate the grid loss of 8760 hours in actual power system. Therefore, the maximum grid loss can be used as a representative to simplify the calculation of system grid loss in most cases.

$$
\begin{aligned}
& E_{\text {LOSSt }}=T_{\max } \cdot \max \left\{\sum_{j=1}^{N_{A}}\left(\gamma_{j} \cdot\left(P_{L j} / U_{j}\right)^{2}\right)\right\} \\
& \eta_{\text {LOSSt }}=E_{\text {LOSSt }} / E_{t r} \times 100 \%
\end{aligned}
$$

where, $\eta_{\text {LOSSt }}$ represents the grid loss rate of the $t$ year, $E_{\text {LOSSt }}$ $E_{t r}$ represent respectively the total grid loss and annual transmission power of the $\mathrm{t}$ year, $\mathrm{kWh} ; T_{\max }$ represents the hours of annual maximum load loss, $\mathrm{h} ; P_{L j}$ represents the active power flow in the $\mathrm{j}$-th line, MW; $U_{j}$ represents the voltage level of the jth line, $\mathrm{kV} ; N_{A}$ represents the set of all the established lines and the lines to be built; $\gamma \mathrm{j}$ represents the resistance of the single line in the jth line, $\Omega$.

2) Voltage qualification rate

The formula of voltage qualification rate is as follows:

$$
\eta_{v q}=\sum_{i=1}^{n} T_{i} / 8760 n
$$

where, $\eta_{v q}$ represents the voltage qualification rate; $T_{i}$ represents the total time (h) when node $i$ runs in the qualified voltage range under one year; $n$ is the total number of nodes.

\section{3) Power supply reliability}

The index of power supply reliability is a comprehensive measurement of the power supply reliability. The system-level reliability index can be derived from the indexes of the load point. The definition and formula of the index are as follows

The system average interruption frequency index SAIFI

$$
S A I F I=\frac{\sum_{i \in R} \lambda_{i} N_{i}}{\sum_{i \in R} N_{i}}
$$

The average power interruption frequency index CAIFI 


$$
C A I F I=\frac{\sum_{i \in R} \lambda_{i} N_{i}}{\sum_{i \in R} M_{i}}
$$

where, $\lambda_{i}$ and $N_{i}$ represent the failure rate of the load node $\mathrm{i}$ and the number of users respectively; $R$ represent $\mathrm{s}$ the set of all load points in the system, and $M_{i}$ is the number of users affected by the power failure of the load node $i$.

\section{Economic Indexes}

\section{1) Power benefits}

The electricity benefits of the distribution network refer to the ratio of the increase electricity generated by the substations and lines after the completion of the project to the project investment. And the annual distribution capacity calculation formula for substations and lines is as follows:

$$
W_{i}=\sum_{i=1}^{2} T_{\max i} * P_{\max i} * \eta_{i}
$$

where, $i=1$ represents the substation, $i=2$ represents the line. $W_{i}$ represents the increase electricity for substations and lines. $T_{\max }$ represents the annual maximum utilization hours, $P_{\operatorname{maxi}}$ represents the maximum load, and $\eta_{i}$ represents the simultaneity rate of maximum load in the power supply range.

2) Benefits of energy conservation and emission reduction

The k-th pollution gas emission $E_{k}(\mathrm{SO} 2, \mathrm{CO} 2, \mathrm{NOx})$ in the system is related to the output and operating characteristics of the unit. $E_{k}$ is calculated as follows:

$$
E_{k}=\sum_{h=1}^{B} P_{g} E_{k g}
$$

where, $P_{g}$ represents the active power of the g-th generator, $B$ represents the number of generators, and $E_{k \mathrm{~g}}$ represents the amount of the k-th pollutant gas released from the unit active power of the g-th generator.

The total amount of pollutant gas $E_{t}$ calculated is as follows:

$$
E_{t}=\sum_{k=1}^{K} z_{k} E_{k}
$$

where, $K$ is the total pollutant gas category; $z_{k}$ is the $\mathrm{k}$-th pollutant gas weight factor. The energy-saving and emissionreducing benefits are the reduction of total pollutant gas emissions.

\section{E. Customer (municipal) Urgent Requirements Index}

By calculating the load rate of the main transformer, the line load rate, and the line's heavy load rate, the weights of the three aspects are obtained by the comprehensive evaluation method and the customer's urgent requirements index is also obtained.

\section{F. Evaluation Index System in Transmission and Distribution Network}

The existing grid project evaluation indexes are evaluated to refine the evaluation indexes needed for the study. The evaluation index systems of reserve projects in distribution network are respectively established according to their different effects in Figure I

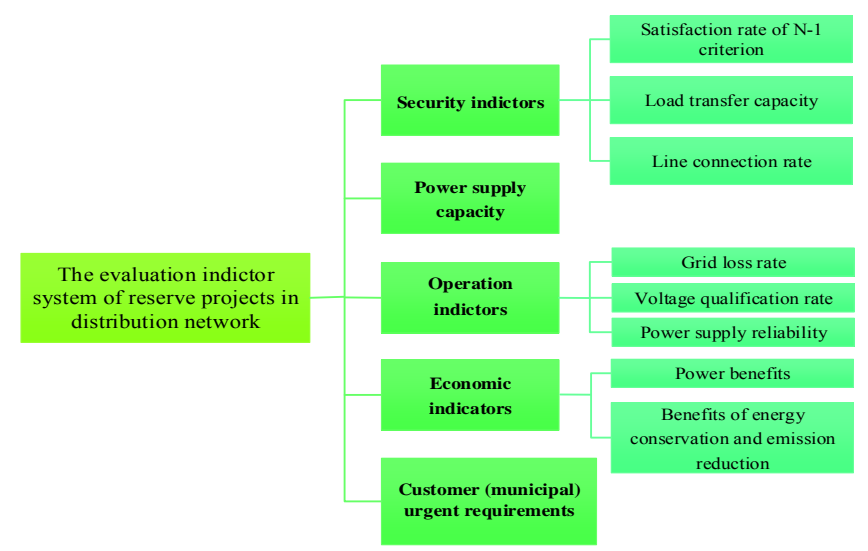

FIGURE I. EVALUATION INDEX SYSTEMS OF RESERVE PROJECTS IN DISTRIBUTION NETWORK

\section{Quantitative Evaluation Method OF GRID RESERVE PROJECTS}

\section{A. The Combination Weighting Method Based on Moment Estimation Theory}

The main idea of the combination weighting method based on the moment estimation theory [10-11]: $d$ samples and q-d samples are extracted from the subjective and the objective weighted population, respectively, and the sample mean and the 2nd-order origin moment are equivalent to the overall expectation and variance. The smaller the deviation between the combined weight $w_{j}$ and the q subjective and objective weights, the better the combined weight $w_{j}$ is. The combined weight $w_{j}$ are solved by the following optimization model:

$$
\left\{\begin{array}{l}
\min H\left(w_{j}\right)=\alpha \sum_{h=1}^{d}\left(w_{j}-w_{h j}\right)^{2}+\beta \sum_{z=d+1}^{q}\left(w_{j}-w_{z j}\right)^{2} \\
\text { s.t. } 0 \leq w_{j} \leq 1,1 \leq j \leq m
\end{array}\right.
$$

The weighting steps of the combination weighting method based on the moment estimation theory: 
1) Using the d subjective weighting methods to weight the evaluation indexes, the subjective weight set for each index is obtained.

$$
W_{H}=\left\{w_{h j} \mid 1 \leq h \leq d, 1 \leq j \leq y\right\}
$$

where, for any $\mathrm{h}, \sum_{j=1}^{y} w_{h j}=1, w_{h j} \geq 0$

2) Using the q-d objective weighting methods to weight the evaluation indexes, the objective weight set for each index is obtained.

$$
W_{z}=\left\{w_{z j} \mid d+1 \leq z \leq q, 1 \leq j \leq y\right\}
$$

where, for any z, $\sum_{j=1}^{y} w_{z j}=1, w_{z j} \geq 0$

3) According to the moment estimation theory, the subjective and objective weight expectation value of each index are respectively calculated.

$$
\begin{gathered}
E\left(w_{h j}\right)=\frac{\sum_{h=1}^{d} w_{h j}}{d}, 1 \leq j \leq y \\
E\left(w_{z j}\right)=\frac{\sum_{z=d+1}^{q} w_{z j}}{q-d}, 1 \leq j \leq y
\end{gathered}
$$

4) The relative importance coefficient $\alpha_{j}$ and $\beta_{j}$ of the subjective and objective weights for each index are respectively calculated.

$$
\begin{aligned}
& \alpha_{j}=\frac{E\left(w_{h j}\right)}{E\left(w_{h j}\right)+E\left(w_{z j}\right)} \\
& \beta_{j}=\frac{E\left(w_{z j}\right)}{E\left(w_{h j}\right)+E\left(w_{z j}\right)}
\end{aligned}
$$

5) The evaluation index in the multi-index decision matrix can be seen as y samples in the population. Therefore, the overall relative importance coefficient of the subjective and objective weights $\alpha$ and $\beta$ can also be obtained.

$$
\alpha=\frac{\sum_{j=1}^{y} \alpha_{j}}{\sum_{j=1}^{y} \alpha_{j}+\sum_{j=1}^{y} \beta_{j}}=\frac{\sum_{j=1}^{y} \alpha_{j}}{y}
$$

$$
\beta=\frac{\sum_{j=1}^{y} \alpha_{j}}{\sum_{j=1}^{y} \alpha_{j}+\sum_{j=1}^{y} \beta_{j}}=\frac{\sum_{j=1}^{y} \beta_{j}}{y}
$$

6) In order to minimize $H\left(w_{j}\right)$ for each evaluation index the model can be optimized as follows.

$$
\left\{\begin{array}{l}
\min H=\left\{H\left(w_{1}\right), H\left(w_{2}\right), \cdots, H\left(w_{y}\right)\right\} \\
\text { s.t. } \sum_{i=1}^{y} w_{j}=1,0 \leq w_{j} \leq 1,1 \leq j \leq y
\end{array}\right.
$$

In order to solve the above model, the multi-objective optimization model is converted into the single-objective optimization model based on the linear weighting method:

$$
\left\{\begin{array}{l}
\min H=\sum_{j=1}^{y} \alpha \sum_{h=1}^{d}\left(w_{j}-w_{h j}\right)^{2}+\sum_{j=1}^{y} \beta \sum_{z=d+1}^{q}\left(w_{j}-w_{z j}\right)^{2} \\
\text { s.t. } \sum_{i=1}^{y} w_{j}=1,0 \leq w_{j} \leq 1,1 \leq j \leq y
\end{array}\right.
$$

By solving the mathematical model of the combination weighting method based on moment estimation theory, the optimal combination weight for each evaluation index can be calculated.

\section{B. Particle Swarm Optimization Algorithm}

Particle swarm optimization algorithm (PSO) [12-14] is an intelligent evolution algorithm based on the behavioral rules of biological populations such as birds. Its optimal value is reached through the collective cooperation between birds. Its optimization formula is as follows:

$$
\begin{aligned}
& v_{I d}^{k+1}=w v_{I d}^{k}+c_{1} r_{\text {and } 1}^{k}\left(p_{\text {bestId }}^{k}-x_{I d}^{k}\right) \\
& +c_{2} r_{\text {and } 2}^{k}\left(g_{\text {bestId }}^{k}-x_{I d}^{k}\right)
\end{aligned}
$$




$$
x_{I d}^{k+1}=x_{I d}^{k}+v_{I d}^{k+1}
$$

where $w$ represents inertia weight, $c_{1}, c_{2}$ represent learning factor and they are usually taken as $c_{1}=c_{2}=2, r_{\text {and } 1}$ and $r_{\text {and } 2}$ represent the random number between $[0,1]$.

\section{Dynamic Sorting Method of GRId Reserve PROJECTS}

All indexes in this paper can be turned into improvement rate indexes for dynamic sorting. The formula is as follows:

$$
V_{i}=\frac{\left|V_{a i}-V_{b i}\right|}{V_{b i}}
$$

where $\mathrm{V}_{i}$ represents the value of improvement rate indexes, $\mathrm{V}_{a i}$ represents the value of improvement rate indexes after the completion of the reserve project, $\mathrm{V}_{b i}$ represents the value of improvement rate indexes before the completion of the reserve project.

The contribution of each index for the transmission and distribution network reserve project is respectively calculated. The formula is as follows:

$$
S_{i k}=V_{i k} / \sum_{k=1}^{M} V_{i k}
$$

where $\mathrm{V}_{i k}$ represents the value of the i-th improvement rate index in the k-th reverse project, $\mathrm{S}_{i k}$ represents the contribution value of each index in the evaluation index system, $M$ represents the number of reserve project.

The specific steps for the dynamic sorting method of reserve projects based on the contribution of unit investment are as follows [15]:

1) The calculation method of the index contribution has been adopted in this paper in order to eliminate the differences between the dimensions and evaluation criteria. the comprehensive contribution of each grid reserve project is obtained by utilizing the value of index contribution and the combination weighting method based on moment estimation theory. The formula is as follows.

$$
S_{k}=\sum_{i=1}^{N} w_{i} S_{i k}
$$

where $S_{k}$ represents the comprehensive contribution value of the k-th reverse project, $\mathrm{S}_{i k}$ represents the contribution value of each index, $N$ represents the number of indexes in the evaluation index system, $w_{i}$ represents the optimal weight vector for each index.
2) Assuming $S_{k}$ represents the comprehensive contribution value of the $k$-th reverse project, the comprehensive contribution value in the unit investment of the $k$-th reverse project $C_{k}$ is calculated as follows:

$$
C_{k}=S_{k} / f_{k}
$$

where $f_{k}$ represents the calculated investment of the $\mathrm{k}$-th reverse project.

The reserve project with the highest contribution in the unit investment is identified as the optimal reserve project in the first round (i.e. the first optimal project is $\left.\operatorname{Max}\left\{C_{1}, C_{2}, \ldots, C_{x}\right\}\right)$

3) For the remaining projects to be selected, repeat steps (1) and (2) to calculate the second optimal project. It should be noted to consider the influence of the projects that have already been optimized on the current grid.

4) For policy projects that are difficult to be included in the quantitative analysis of the above models, the manual interventions are added by the experts to advance or delay the order of the reserve projects.

5) The steps (3) and (4) are repeated until the sorting of all reserve projected are arranged:

6) Check whether all weak points of the current power grid are resolved after all construction projects are completed. If not completely resolved, the suggestions and opinions can be provided to the higher-level power grid to appropriately increase the corresponding engineering projects.

\section{CASE StUdy}

\section{A. Example Discription}

Based on the indexes definition of the evaluation system and its calculation method, the relevant actual data are determined as follows:

According to the distribution network project constructed in a certain region, the number of main transformers in the area is 36 , the total capacity is 1134 MVA, the number of lines is 279 , the number of lines that can pass the "N-1" check is 97 , and number of the connection lines between stations is 84 . The load transfer capacity is $256 \mathrm{MW}$; the grid loss rate is $28 \%$; the voltage qualification rate is $73 \%$; the power supply reliability is 0.16 times / user; the amount of pollutant emission is $61.2 \mu \mathrm{g} / \mathrm{m}^{3}$; the power supply capacity is $910 \mathrm{MVA}$, the load is $750 \mathrm{MW}$, the power grid asset is 1.2 billion yuan, the main transformer load rate is $52 \%$, the line load rate is $76 \%$, and the line heavy load rate is $32 \%$.

\section{B. Calculation Results and Analysis}

Due to limited space, the results of each index are ignored. However, the index contribution value of the reverse project can be shown in Table I. In this case, the subjective weighting method in this paper refers specifically to the Delphi method and the AHP; the objective weighting method specifically refers to the entropy method and the mean square difference 
method. The weight results of the reverse projects are shown in the following Table II.

TABLE I. INDEX CONTRIBUTION VALUE OF THE REVERSE PROJECT

\begin{tabular}{|c|c|c|c|c|}
\hline \multicolumn{2}{|c|}{ Index system } & \multicolumn{3}{c|}{ Index contribution } \\
\cline { 2 - 4 } Security indexes & Satisfaction rate of N-1 criterion & project A & project B & project C \\
\cline { 2 - 4 } & Load transfer capacity & $51.9 \%$ & $39.8 \%$ & $28.3 \%$ \\
\cline { 2 - 4 } & Line connection rate & $23.9 \%$ & $41.6 \%$ & 0 \\
\hline \multirow{2}{*}{ Power supply capacity } & 930 & $25.2 \%$ & $50.9 \%$ \\
\hline \multirow{3}{*}{ Operation indexes } & Grid loss rate & $21.3 \%$ & 930 & $11.8 \%$ \\
\cline { 2 - 5 } & Voltage qualification rate & $19.7 \%$ & $39.3 \%$ & $39.3 \%$ \\
\cline { 2 - 5 } & Power supply reliability & $28.5 \%$ & $40.1 \%$ & $40.1 \%$ \\
\hline \multirow{3}{*}{ Economic indexes } & Power benefits & $29.8 \%$ & $36.8 \%$ & $33.5 \%$ \\
\cline { 2 - 5 } & $\begin{array}{c}\text { Benefits of energy conservation } \\
\text { and emission reduction }\end{array}$ & $29.9 \%$ & $36.3 \%$ & $33.7 \%$ \\
\hline \multicolumn{2}{|c|}{ Customer (municipal) urgent requirements index } & $30.2 \%$ & $30.2 \%$ & $32.6 \%$ \\
\hline
\end{tabular}

TABLE II. THE WEIGHT CALCULATION RESULTS

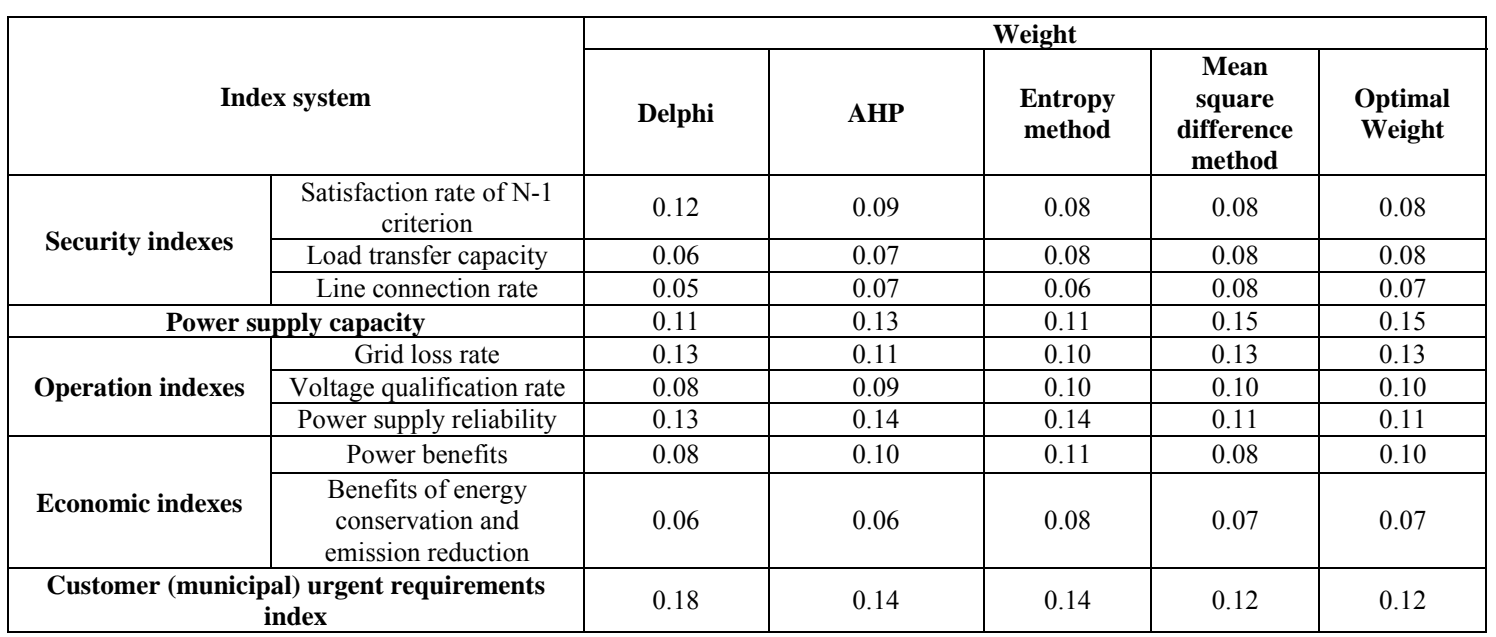

The relative importance degree of subjective and objective weights is calculated as $\alpha=0.515$ and $\beta=0.485$. Then, the comprehensive contribution value in the unit investment of the reverse project are shown in Table III.

TABLE III. COMPREHENSIVE CONTRIBUTION VALUE IN THE UNIT INVESTMENT OF THE REVERSE PROJECT

\begin{tabular}{|c|c|c|c|}
\hline Project & Project A & Project B & Project C \\
\hline $\begin{array}{c}\text { Comprehensive contribution } \\
\text { value }\end{array}$ & 0.273 & 0.410 & 0.327 \\
\hline $\begin{array}{c}\text { Construction investment } \\
\text { (million yuan) }\end{array}$ & 1.85 & 2.18 & 2.07 \\
\hline $\begin{array}{c}\text { Comprehensive contribution } \\
\text { value in the unit investment }\end{array}$ & 0.148 & 0.188 & 0.157 \\
\hline
\end{tabular}

The optimization of the grid reserve project is based on its contribution to the unit capital. The reserve project will be given priority if it has a large contribution to the unit funds. Otherwise, it will delay construction. As shown in Table V, Project $\mathrm{B}$ will be given priority and Project A, C will be delayed.

In this example, to determine the construction sequence of $\mathrm{A}$ and $\mathrm{C}$ projects, it is necessary to sort dynamically the reserve projects and recalculate the contribution of indexes in
$\mathrm{A}$ and $\mathrm{C}$ projects. The contribution of indexes under unit funds, as shown in the Table IV:

TABLE IV. CONTRIBUTION OF INDEXES IN A AND C PROJECTS

\begin{tabular}{|c|c|c|}
\hline Project & Project A & Project C \\
\hline $\begin{array}{c}\text { Comprehensive contribution } \\
\text { value }\end{array}$ & 0.451 & 0.549 \\
\hline $\begin{array}{c}\text { Construction investment } \\
\text { (million yuan) }\end{array}$ & 1.85 & 2.07 \\
\hline $\begin{array}{c}\text { Comprehensive contribution } \\
\text { value in the unit investment }\end{array}$ & 0.243 & 0.265 \\
\hline
\end{tabular}

Therefore, thought the dynamic order of the reserve projects the final $\mathrm{A}, \mathrm{B}$, and $\mathrm{C}$ project construction sequence is as follows: $\mathrm{B}$ project $-\mathrm{C}$ project- $\mathrm{A}$ project.

\section{CONCLUSION}

1) In this paper, a scientific and rational reserve project evaluation index system is put forward. The index system includes five indexes, measuring the contribution of reserve projects on the grid from five different aspects. Through the calculation methods of each index and the quantitative evaluation method, the comprehensive score of each reserve project is obtained. 
2) The degree of improvement of each evaluation index is calculated by analyzing the improvement of the entire grid indexes in each grid reserve project. Through the weight study of various indexes, a combination weighting method based on moment estimation theory is proposed in this paper.

3) An optimal sorting model for grid reserve projects is established. The optimal sorting method of grid reserve projects is finally proposed, considering the comprehensive contribution of grid reserve projects and the investment of the reserve project.

\section{ACKNOWLEDGMENT}

This work was support by State Grid Corporation of China (Project Name: Research on Power Grid Precision Investment Decision-making Technology Based on Investment Capability Constraints and Multi-link Coordination).

\section{REFERENCES}

[1] Chai Zhonghua, Zheng Chuiyong, and Cai Hua. "Risk Assessment Index System Construction of Venture Capital Projects". Statistics and Decision. J. 2010(08), pp: 176-178.

[2] Chai Zhonghua, Zheng Chuiyong, and Cai Hua. "A New Method of Venture Capital Evaluation". Statistics and Decision.J. 2010(05), p.162164.

[3] Chai Zhonghua. Post-evaluation theory, method and application of power project [D]. Hohai University, 2007.

[4] Zheng Yan. Post-evaluation research and application of power investment projects [D]. North China Electric Power University (Hebei), 2007.

[5] Zhang Jian, Zheng Yan, WEN Weining, Zhao Zhe, Wang Yaping, Chen Li. A review of power grid engineering cost management during the 11th Five-Year Plan period. Energy Technology, J, 2012, 24(03),pp: 4451.

[6] Liu Wei, Guo Zhizhong. Research on the Safety Index of Distribution Network. Proceedings of the CSEE, J. 2003, 23(8), pp:85-90.

[7] Li Zhongcheng, Zhang Buhan, Duan Yao. Probability Available Power Transmission Capability of Power System with Large Scale Wind Farms. Proceedings of the CSEE, J. 2014, 34(4), pp:505-513.

[8] Li Mengdi, YAN Siqing, GAO Mengya, ZHANG Jing, Geng Chenxi. Grid Risk Level Evaluation System Based on Moment Estimation Theory. Science and Technology Wind, J. 2017(13), pp: 176-178.

[9] Xue Liming, Su Chao, Cui Chaoqun, Sun Yanding. Application of Optimal Combination Weighting Method under Moment Estimation Theory in the Evaluation of Mineral Resources. China Mining Magazine, J. 2017, 26(04), pp: 41- 46.

[10] Chen Hongwei, Xu Qian, Zheng Xiao, Lan Zhou. Comprehensive coordination evaluation of power grid planning scheme based on combination weighting method[J]. Zhejiang Electric Power,2014,33(09): 1-5+21.

[11] Lu Zhigang, Han Yanling, Chang Lei. Economic evaluation of distribution network operation based on combined weight. Power System Protection and Control, J.2008, 36(18), pp:1-5.

[12] Tian Ye. Particle Swarm Optimization Algorithm and Its Application[D]. Jilin University, 2010.

[13] Zhao Naigang, Deng Jingshun. A Review of Particle Swarm Optimization Algorithm. Science and Technology Innovation Herald, J. 2015, 12(26), pp: 216-217.

[14] Jiang Hao. Research on particle swarm optimization based on multiobjective optimization [D]. Xiangtan University, 2006.

[15] Li Canbing, Fu Meiping, Liang Jinzhao. Optimal scheduling method for power grid construction projects based on network coordination evaluation. Power System Protection and Control, J. 2010, 38(17), pp:112-115. 